\section{Nutzen Sie Placeboeffekte!}

\author{
Placeboeffekte lassen sich durch psychologische und \\ neurobiologische Mechanismen erklären, ihre Wirksam- \\ keit ist wissenschaftlich bewiesen. Machen Sie sich diese \\ Mechanismen in Ihrem Praxisalltag zunutze. Besonders \\ Schmerzpatienten werden davon profitieren!
}

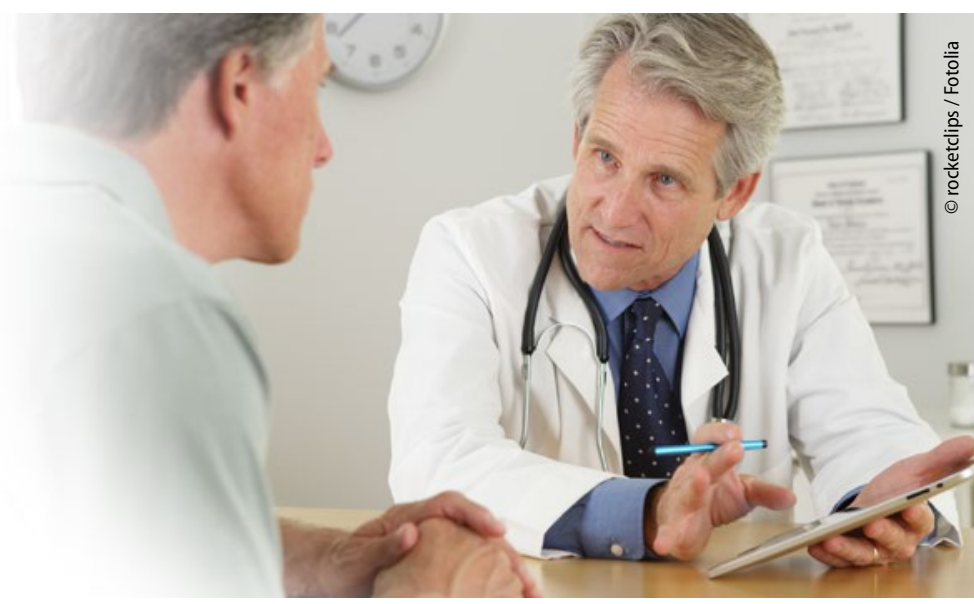

_ Unter Placeboeffekten versteht man positive physiologische und psychologische Veränderungen nach der Einnahme von Medikamenten ohne spezifischen Wirkstoff oder nach Scheineingriffen. „Wir wissen heute, dass auch neuropsychologische Phänomene wie Erwartungshaltung der Patienten bzgl. der Wirkung einer Therapie, assoziative Lernprozesse und die Qualität der ArztPatienten-Beziehung für den Placeboeffekt entscheidend sind“, erläuterte Prof. Ulrike Bingel, Essen. Diese Prozesse induzieren komplexe psycho-neurobiologische Phänomene im ZNS, die wiederum peripher-physiologische Abläufe und Endorganfunktionen modulieren.

\section{Erwartung verspricht Erfolg}

Der z.T. sehr ausgeprägte Benefit von Placebobehandlungen selbst im Vergleich zu einer medizinischen Standardbehandlung konnte in der GERAC-Akupunktur-Studie, einer großen kontrollierten, randomisierten Untersuchung bei 1.000 Patienten mit chronischen Rückenschmerzen, dokumentiert werden. Es ergab sich kein Unterschied zwischen der Verum- und der Scheinakupunktur, aber ein signifikanter positiver Unterschied für beide Akupunkturstrategien gegenüber der medizinischen Standardbehandlung. „Die Studie zeigt, dass eine Scheinbehandlung durchaus wirksam sein kann, wenn der Patient eine Wirksamkeit erwartet", so Bingel. Wer an etwas glaubt, dem hilft es auch.

\section{Lernen beeinflusst die Wirkung}

Auch Lernvorgänge gehören $\mathrm{zu}$ den Schlüsselmechanismen von Placebo-, aber auch Noceboantworten. So führt die wiederholte Assoziation eines neutralen Stimulus wie Aussehen oder Geschmack einer Tablette mit der pharmakologischen Wirkung der Tablette zu einer „konditionierten“ Reaktion, d.h. nach wiederholter Einnahme der Tablette kann die Wirkung auch durch eine wirkstofffreie Tablette ausgelöst werden. Solche konditionierten Effekte konnten auch nach wiederholter Gabe von intranasalem Insulin im Hinblick auf die körpereigene Insulinsekretion bzw. den Blutzuckerspiegel nachgewiesen werden.

\section{Placeboanalgesie}

Der Einfluss kognitiv-emotionaler Faktoren auf die zentrale Schmerzwahrnehmung bzw. -modulation und die spinale Schmerzverarbeitung und somit auf die Schmerzchronifizierung ist heute gut belegt, d.h. kognitiv-emotionale Faktoren können die Schmerzleitung im Rückenmark hemmen oder begünstigen. "In die Placeboantworten sind dieselben physiologischen Systeme involviert, die auch Angriffspunkte pharmakologischer Therapien sind“, so Bingel.

\section{Placeboeffekte in der Praxis}

Aus ethischen und juristischen Gründen verbietet sich die Gabe „reiner" Placebos, wenn der Patient nicht über das Wesen der Behandlung informiert ist. „Doch die zugrundeliegenden Placebomechanismen wie Erwartung und Lernen und auch eine gezielte Arzt-Patienten-Kommunikation sollten genutzt werden, um die Wirkung von pharmakologischen und anderen Behandlungen zu optimieren“, empfahl Bingel. Der Einsatz solcher
Ob eine Therapie wirkt, hängt auch von der ärztlichen Zuwendung ab.

„Placebomechanismen“verletze keinerlei ethische Grenzen. Dazu gehören:

- Patientenverständliche Aufklärung über Erkrankung und Behandlung

- Formulierung individueller Therapieziele

- Vermeiden unrealistischer Erwartungen

- Berücksichtigung individueller Präferenzen bei der Behandlung

- Kopplung der medikamentösen Therapie mit sensorischen Ereignissen (Gefühl, Geschmack, Geruch)

- Kombination von Medikamenten mit nicht-medikamentösen Maßnahmen wie z. B. Entspannungstechniken bei der Schmerztherapie

- Behandlung der Komorbiditäten Angst bzw. Depression

- Empathische und authentische ArztPatienten-Kommunikation.

\section{FAZIT FÜR DIE PRAXIS}

1. Placeboeffekte basieren auf Erwartungen und Lernvorgängen.

2. Sie spielen eine große Rolle bei der Therapie von Patienten mit chronischen Schmerzen.

3. Sie sind involviert in physiologische Systeme, die auch Angriffspunkt pharmakologischer Therapien sind.

Dr. Peter Stiefelhagen

- Quelle: Praxis update, 29.4.2016 in Köln 